\title{
Autocollimators for Deflectometry: Current Status and Future Progress
}

\author{
Ralf D. Geckeler ${ }^{1}$, Andreas Just ${ }^{1}$, Michael Krause ${ }^{1}$, and Valeriy Yashchuk ${ }^{2}$ \\ ${ }^{1}$ Physikalisch-Technische Bundesanstalt Braunschweig und Berlin (PTB) \\ Bundesallee 100, D-38116 Braunschweig, Germany \\ ${ }^{2}$ Advanced Light Source, Lawrence Berkeley National Laboratory, \\ 1 Cyclotron Road, Berkeley, CA 94720-8199, USA \\ Email:Ralf.Geckeler@ptb.de \\ Phone: +49-531-592-5220, Fax: +49-531-592-695220
}

\section{Introduction}

The proliferation of autocollimator-based surface profilometers at synchrotron metrology laboratories worldwide necessitates a detailed understanding of the parameters influencing their angular response. A comprehensive overview of the current status of autocollimator characterization and calibration at the Physikalisch-Technische Bundesanstalt (PTB) and its implications for their optimal application are provided, as well as information on future challenges and expected progress. Autocollimator-baseddeflectometric profilometers are in operation at the PTB, the Advanced Light Source (ALS), Lawrence Berkeley National Laboratory, USA, the BESSY II, Helmholtz-Zentrum Berlin für Materialien und Energie GmbH, Germany, and the Diamond Light Source Ltd (DLS), UK.

\section{Current Status}

A continuous topic of research at the PTB is to investigate the factors influencing the angular response of autocollimators and to provide the most accurate calibration of the instruments to aid users in their application and manufacturers in their improvements [1-3]. At the PTB, the calibration of autocollimators is realized by a direct comparison of the devices with the primary angle reference standard [1]. With highly stable autocollimators, calibrations with standard measurement uncertainties of $u=0.003$ arcsec (15 nrad) have been achieved [2,3]. The influence of a number of parameters on the angle measurement with autocollimators have been investigated in detail, such as the reflectivity of the surface under test (SUT), the diameter and shape of the aperture stop, its position both along the autocollimator's optical axis and perpendicular to it [2], as well as the optical path length of the autocollimator beam [3]. Extensive information on these errors and their minimization will be presented.

\section{Future Needs and Developments}

Progress in the following areas is needed: (1) the extension of the angle calibration of autocollimators from the plane to the solid angle, (2) the characterization of the influence of the varying optical path length between the autocollimator and the SUT, (3) the reduction of the feasible aperture size and minimization of the resulting angle errors, (4) the improvement of the algorithms for locating the reticle image on the autocollimator's CCD detector, and (5) the characterization of the influence of the SUT curvature.

Item (1): Beam-shaping optical surfaces in synchrotrons are often highly rotationally asymmetric which leads to large deflections of the surface-probing autocollimator beam perpendicular to the main profiling direction. Therefore, it is necessary to extend the angle calibration of 
autocollimators from the plane to the solid angle, i.e., to obtain 2D calibration in two axes. Developments at PTB in this field are presented.

Item (2): The further characterization of the influence of the optical path length between the autocollimator and the SUT on its angular response is necessary as most deflectometers use a moving pentaprism to scan the surface under test which induces large changes in this parameter. Information on a planned testing device is provided.

Item (3): Small (mm-sized) autocollimator beam apertures are needed for high spatial resolution which introduce (due to diffraction and interference) additional angle errors. Information on a collaboration to improve autocollimator performance at small apertures by introducing phase shifting reticles is presented.

Item (4): In addition to angle errors on long angular scales, CCD-based autocollimators show periodic errors at small (a few arcsec) scales which correspond to the detector's pixel size. Detailed information on them is presented. The improvement of the algorithms for locating the reticle image on the autocollimator's CCD detector with sub-pixel resolution to suppress periodic angle errors would be helpful.

Item (5): The strong (and locally varying) curvature of most synchrotron beamline optics affects both the location and the quality of the image of the autocollimator's reticle on its CCD detector. Apart from first measurements performed at PTB, this important effect has not yet been investigated in detail. A systematic effort is necessary, e.g., with the UTM (Universal Test Mirror) system built at the ALS [4].

\section{Acknowledgements}

The Advanced Light Source is supported by the Director, Office of Science, Office of Basic Energy Sciences, Material Science Division, of the U.S. Department of Energy under Contract No. DE-AC02-05CH11231 at Lawrence Berkeley National Laboratory.

\section{Disclaimer}

Certain commercial equipment, instruments, or materials are identified in this document. Such identification does not imply recommendation or endorsement by the US Department of Energy, LBNL, or ALS, nor does it imply that the products identified are necessarily the best available for the purpose.

\section{References}

[1] A. Just, M. Krause, R. Probst, and R. Wittekopf, Metrologia 40 (2003) 288-294

[2] R.D. Geckeler and A. Just, Proc. SPIE 6704 (2007) 670407/1-12

[3] R.D. Geckeler and A. Just, Proc. SPIE 7077 (2008) 70770B/1-12

[4] V.V. Yashchuk et al., Proc. SPIE 6704 (2007) 67040A/1-12 